\title{
The Interactions of Small Proline-Rich Proteins with Late Cornified Envelope Proteins are Involved in the Pathogenesis of Psoriasis
}

\author{
Siyu Tian \\ Shuming Chen \\ Yongyi Feng \\ Yong $\mathrm{Li}$
}

School of Life Sciences, Xiamen University, Xiamen, People's Republic of China
Correspondence: Yong $\mathrm{Li}$

School of Life Sciences, Xiamen

University, Xiamen, Fujian, 361005,

People's Republic of China

Tel +86-592-2182510

Fax +86-592-2182560

Email yongli@xmu.edu.cn
Purpose: Psoriasis is a common cutaneous disease with multiple characteristics including inflammation and aberrant keratinocyte proliferation. However, the pathogenesis of psoriasis is not completely clear yet. The objective of this study is to perform an in-depth analysis of the association between SPRR and LCE in the pathogenesis of psoriasis.

Methods: In this study, we explore the differentially expressed genes (DEGs) in psoriasis by analyzing different gene expression profiles obtained from the Gene Expression Omnibus (GEO) database. The DEGs were examined using gene ontology (GO) functional enrichment analysis and protein-protein interactions (PPI) network. Correlation analysis in $\mathrm{R}$ studio software was used to analyze the association between SPRR and LCE genes. Further, potential direct protein-protein interactions between SPRR proteins and LCE3D were verified by co-localization observations and co-immunoprecipitation (CO-IP) assays in 293T cells. Also, the expression levels of SPRR and LCE genes were detected in lesional skin of the IMQ-induced psoriasiform dermatitis mice using RT-PCR.

Results: Interestingly, the small proline-rich (SPRR) and late cornified envelope (LCE) genes were identified as a module in the constructed PPI network. And the analysis of the gene expression profile GSE63684 showed that both SPRR family and LCE family genes were significantly upregulated in imiquimod (IMQ) induced psoriasiform dermatitis mice. Also, the correlation analysis in $\mathrm{R}$ studio software recognized the association of SPRR and LCE genes, which were further verified by co-localization and co-immunoprecipitation (COIP) assays in 293T cells, and the results show that the direct interactions between SPRR2 and LCE3D. Notably, we also found that the expression levels of SPRR and LCE genes were significantly increased in the IMQ-induced psoriasiform dermatitis mice, while specifically decreased under the tazarotene cream treatment, indicating that the SPRR and LCEs were regulated simultaneously in psoriasis.

Conclusion: In summary, our study found that interactions between SPRR proteins and LCE proteins may provide new insights into the pathogenesis of psoriasis.

Keywords: psoriasis, bioinformatics analysis, interaction, SPRR, LCE

\section{Introduction}

Psoriasis is a common chronic inflammatory skin disease, prevalent in $2-4 \%$ of worldwide population. $^{1-3}$ It is not purely a skin disorder and may also cause other comorbidities. $^{4,5}$ It is currently believed that environmental factors, infections, genetic factors and immune disorders may induce this disease. ${ }^{6}$ However, the pathogenesis of psoriasis remains unclear yet. Therefore, it becomes important to explore the possible pathogenesis of psoriasis. 
Recently, some studies have found that the interactions between genes are associated with the pathogenesis of psoriasis. For example, the gene-gene interactions in IL23/Th17 pathway contribute to psoriasis susceptibility in Chinese Han population. ${ }^{7,8}$ Specifically, the interactions between late cornified envelope proteins and corneodesmosin were also analyzed. ${ }^{9}$ Additionally, stepwise regression analysis found that LILRB2 binds to HLA-B in two large psoriasis genome-wide association study cohorts, with a total of 10,069 subjects. $^{10}$

Previous studies have reported that psoriasis is generally caused by epidermal hyperproliferation and aberrant keratinocyte differentiation. ${ }^{11,12}$ Relatedly, Epidermal Differentiation Complex (EDC) is a gene cluster on human chromosome 1q21, which comprises the genes encoding structural and regulatory proteins crucial to keratinocyte differentiation and stratum corneum functions. ${ }^{13,14}$ Among genes in EDC, two gene families encoding small proline-rich protein (SPRR) and late cornified envelope (LCE) protein are important components of cornified cell envelope. ${ }^{15,16}$ Thus, SPRR and LCE genes were identified as risk factors for psoriasis patients. ${ }^{17,18}$ However, the association of SPRR and LCE genes has not yet been performed in-depth analysis in psoriasis.

Bioinformatics analysis of gene expression profiles has become an important method to investigate the pathogenesis of complex diseases in recent years. ${ }^{19,20}$ In our study, we downloaded the gene expression profile GSE109248 from the Gene Expression Omnibus database and used limma packages in R studio software to explore the differentially expressed genes (DEGs) in psoriasis, which were further analyzed by gene ontology (GO) enrichment analysis and protein-protein interaction (PPI) network. Interestingly, SPRR and LCE genes, which are associated with keratinocyte differentiation, were identified as a module in the constructed PPI network. In addition, an analysis of the gene expression profile GSE63684 showed that both SPRR and LCE genes were significantly upregulated in IMQ-induced psoriasiform dermatitis mice. Besides, the association of SPRR and LCE genes was detected by correlation analysis in $\mathrm{R}$ studio software. We then investigated potential direct protein-protein interactions between SPRR proteins and LCE3D. Colocalization of SPRR2 and LCE3D were observed in 293T cells by fluorescence microscopy. Moreover, the coimmunoprecipitation (CO-IP) results provided the evidence for direct interactions between SPRR2 and LCE3D. Subsequently, a model of IMQ-induced psoriasiform dermatitis mice was constructed. We found that the expression levels of SPRR and LCE genes were significantly increased in the IMQinduced psoriasiform dermatitis mice, while decreased in tazarotene cream treated mice, indicating that the SPRR and LCEs were regulated simultaneously in psoriasis. Consequently, the interactions of SPRR proteins with LCE proteins may provide new insights into the pathogenesis of psoriasis.

\section{Materials and Methods \\ Gene Microarray Data}

Gene microarray data GSE109248 were downloaded from GEO database of the National Center Biotechnology Information (NCBI), ${ }^{21,22}$ which was executed with GPL10558 Illumina Human HT-12 V4.0 expression beadchip. GSE109248 contains 14 controls and 17 psoriasis from formalin fixed, paraffin-embedded human skin samples. GSE63684 consists of three control mice and two mice with Imiquimod-induced psoriasis based on GPL6246 Platforms (Affymetrix Mouse Gene 1.0 ST Array).

\section{Identification of DEGs}

GSE109248 was processed using GEO query and limma $\mathrm{R}$ package from the Bioconductor project. Subsequently, the DEGs were identified between psoriasis samples and control samples based on the cutoff criterion, $\mid \log 2$ fold change (FC) $\mid>2.5$ and $\mathrm{P}$ values $<0.05$. Volcano Plot and heatmap were generated by ggplot2 and pheatmap package in R studio software. Correlation analysis was conducted with ggstatsplot and ggcorrplot package, and Pearson correlation coefficient was calculated.

\section{Gene Ontology Enrichment and KEGG Pathway Analysis}

The Database for Annotation, Visualization and Integrated Discovery (DAVID) is an online gene analysis tool that provides comprehensive annotations of gene functions and enrichment analysis, DAVID 6.8 was used for Gene Ontology (GO) and KEGG pathway enrichment analysis of DEGs. ${ }^{23}$ And $\mathrm{P}<0.05$ was considered as statistical significance.

\section{PPI Network Construction and Module Analysis}

STRING is an online tool for known and predicted protein-protein interactions. ${ }^{24}$ We put the DEGs into the STRING (Search Tool for the Retrieval of Interacting Genes) online database and then PPI networks were 
constructed, in which the score $\geq 0.4$ was set as the cutoff threshold. ${ }^{25}$ Then, the PPI networks were visualized using Cytoscape software, and MCODE (Molecular Complex Detection) was further applied to establish module of DEGs. ${ }^{26}$ Degree $>10$ and $\mathrm{P}$ values $<0.05$ are used as the cutoff criterion.

\section{Animal Procedures}

All the animal experiments were approved by the Animal Ethics Committee of Xiamen University (acceptance no. XMULAC20170313). All animal experiments were performed in compliance with the guidelines from the Institutional Animal Care and Use Committee at Experimental Animal Centre in Xiamen University. Female BALB/c mice (6-8 weeks old) were purchased from Beijing Vital River Laboratory Animal Technology Co., Ltd. The mice were housed under temperature $\left(22 \pm 2^{\circ} \mathrm{C}\right)$ and humidity $(50 \pm 20 \%)$ condition with a standard light $(12 \mathrm{~h}$ light/ dark) cycle. The BALB/c mice were randomly divided into 3 groups, and the mice had their hair removed with veet hair removal cream on the back (area about $2 \times 2 \mathrm{~cm}$ ). In the control group $(n=5)$, mice were treated with vaseline cream. In the IMQ group $(n=8)$, psoriasis was induced by IMQ treatment, as previously reported. ${ }^{27,28}$ Briefly, 5\% IMQ cream ( $62.5 \mathrm{mg}$ per mouse) was applied evenly every day. In tazarotene cream treatment group $(\mathrm{n}=8)$, mice were treated with tazarotene cream two hours after applying IMQ cream. Mice were killed after 6 days; tissues were collected and frozen in liquid nitrogen for use. H\&E staining was performed according to standard procedures.

\section{mRNA Isolation and Quantitative Real-Time PCR}

Total RNA was isolated from tissues using RNA kit (Omega Bio-Tek, GA), and cDNA was obtained using TAKARA reverse transcription kit. Quantitative real-time PCR was performed on a CFX96 Real-Time PCR Detection System (Bio-Rad). Relative mRNA expression levels were normalized to GAPDH levels. The primers used in this assay were listed in the Table S4.

\section{Plasmid Construction and Coimmunoprecipitation}

The cDNA encoding murine SRPP2A, SPRR2F and LCE3D were obtained from murine skin RNA using RTPCR. Subsequently, full-length fragments with BglII and XhoI enzyme recognition sites were amplified by polymerase chain reaction and subcloned into pCMV HA and Flag fusion vector. The constructs were confirmed by sequencing.

For coimmunoprecipitation assay, 293T cells were maintained in DMEM containing 10\% fetal bovine serum and were transiently transfected using Lipofectamine 2000 (Invitrogen). Before 24 hours of transfection, cells were seeded on $60-\mathrm{mm}$ plates. 293T cells were transfected with the above constructed plasmids. After 24 hours, cells were collected, followed by immunoprecipitation. Western blotting was performed with anti-HA (Santa Cruz Biotechnology) and anti-Flag (Sigma).

\section{Fluorescence Microscopy}

293T cells were transiently transfected with GFP-tagged SPRR2A or SPRR2F and mRuby2-tagged LCE3D plasmids. After $24 \mathrm{~h}$ of transfection, 293T cells were fixed with $4 \%$ paraformaldehyde and permeabilized with $0.5 \%$ Triton X-100 in PBS. Cell nuclei were stained with DAPI before images captured by a Leica SP8 laser-scanning confocal microscope (Leica). Images were processed with LAS X software (Leica).

\section{Results \\ Identification of DEGs}

In present study, we analyzed the GSE109248 dataset including 17 patients with psoriasis and 14 healthy controls. There are 131 DEGs identified in the GSE109248 using the limma package based on the criteria of $P$ values $<0.05$ and $\mid \log 2$ fold change (FC) $\mid>2.5$, are described by volcano plot and heatmap (Figure 1A and B). Among them, there were 86 upregulated and 45 downregulated genes (Table S1).

\section{Gene Ontology Function Enrichment Analysis}

In order to clarify the biological functions performed by these DEGs, GO functional annotation and pathway enrichment analyses were conducted in DIVID online database. $\mathrm{P}<0.05$ is considered to be statistically significant. As presented in Figure 2 and Table S2, DEGs were classified into functional terms of biological process (BP) and molecular function (MF). In BP group, the DEGs were significantly enriched in keratinocyte differentiation, keratinization, peptide cross-linking and epidermis development. In MF group, they were primarily involved in the structural molecule activity and cytokine activity. 
A

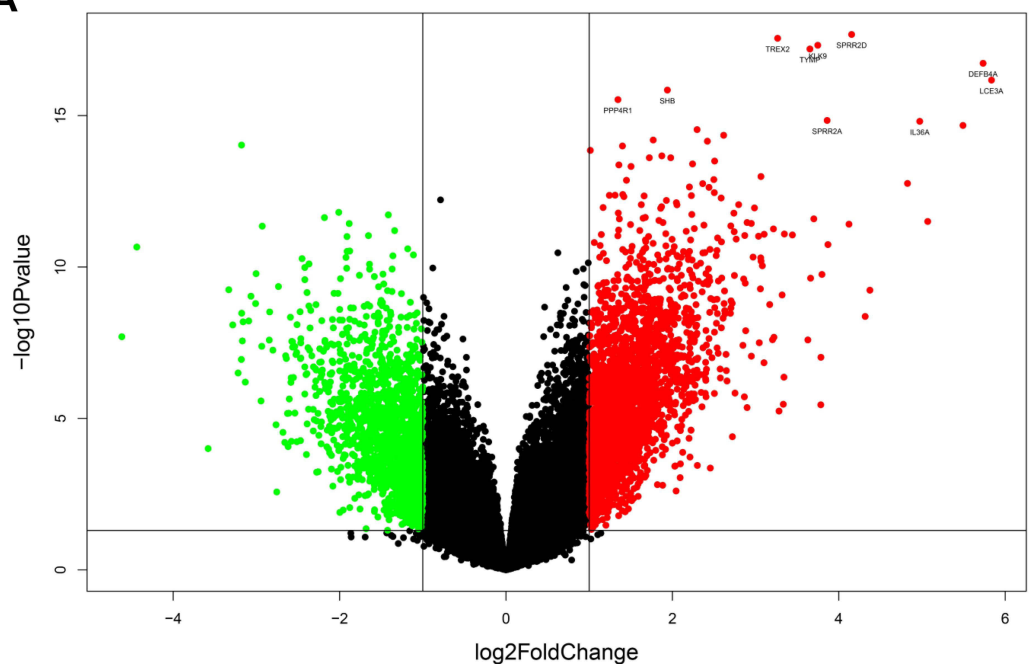

B

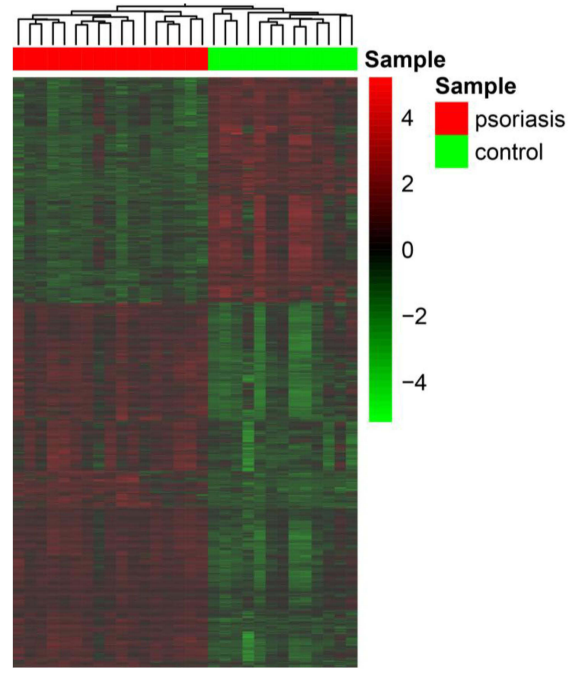

Figure I Identification of DEGs between psoriasis patients and healthy controls. (A) Volcano plot of DEGs. Red points represent up-regulated genes; green points represent down-regulated genes. (B) The heatmap of DEGs. Red colors indicate up-regulated genes and green colors indicate down-regulated genes.
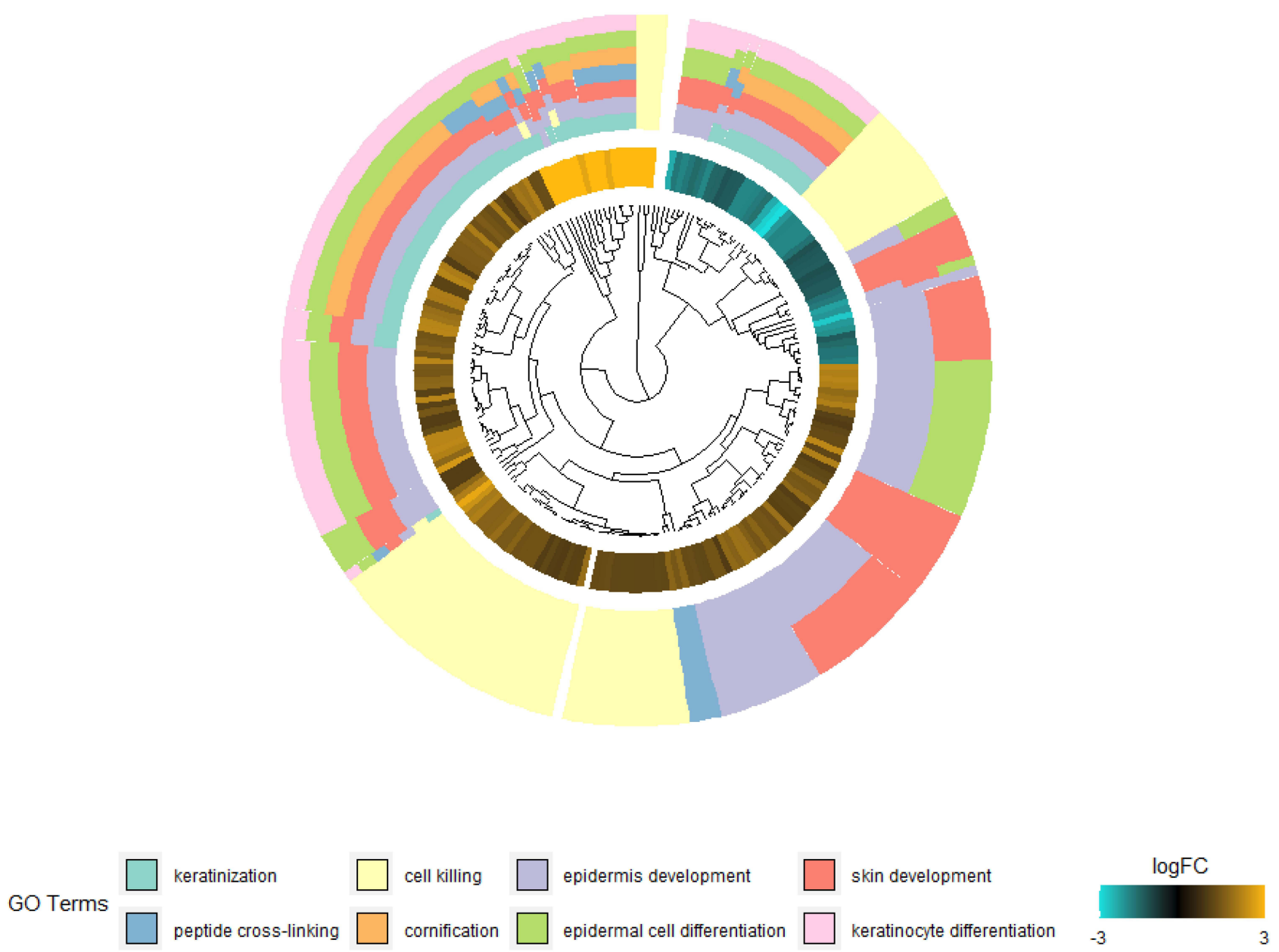

Figure 2 Gene ontology enrichment analysis of DEGs in psoriasis patients. 
Subsequently, we analyzed up-regulated DEGs, which were mainly involved in keratinocyte differentiation, keratinization and structural molecule activity (Table S3).

\section{Module Analysis and Hub Genes Identification from PPI Network}

In order to identify key modules and hub genes, 131 DEGs were uploaded to the STRING database. Then, PPI network was established in Cytoscape software, including 124 nodes and 186 edges, and 7 genes were failed into the PPI network (Figure 3A). The top hub genes PI3, S100A7, SPRR1B, CXCL8, SPRR3, SPRR2G, LCE3D, SPRR2B, SPRR2D, SPRR2A and SPRR2F were identified by network analyzer. Furthermore, a significant module was analyzed based on the Cytoscape software APP MCODE. Surprisingly, the module consists mainly of SPPR and LCE family genes (Figure 3B).

\section{The SPPR and LCE Genes Expression in Mice and GO Enrichment Analysis Based on GEO Dataset}

Additionally, the GEO dataset GSE63684 was analyzed. Consistent with previous results, the expression level of SPPR and LCE genes was significantly upregulated in

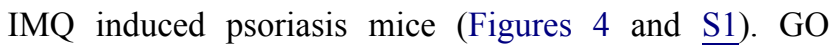
enrichment analysis showed that the SPPR and LCE genes were also significantly enriched in keratinocyte differentiation, keratinization, peptide cross-linking, epidermis development, cornified envelope and structural molecule activity (Figure 4C).

\section{Interaction Analysis of SPRR with LCE}

It has been reported that SPRR and LCE protein family genes formed an Epidermal Differentiation Complex (EDC). ${ }^{13,29,30}$ However, the association between the SPRR and LCE genes is still unclear. Subsequently, bioinformatics analysis was executed in $\mathrm{R}$ studio software (Figures 5A and $\underline{\mathrm{S} 3}$ ). We first found a strong correlation between LCE3D and SPRR2A or SPRR2F genes. Further, the whole LCE family genes have distinct correlation with those of SPRR (Figure 5B). Then, colocalization of SPRR2 and LCE3D was observed in cells using fluorescence microscopy by co-expression of GFP-SPRR2A or GFP-SPRR2F and mRuby2-LCE3D proteins in 293T cells (Figure 5C). To further verify their interaction, HA-tagged or Flag-tagged LCE3D, HA-tagged SPRR2A and HA-tagged or Flag-tagged
SPRR2F plasmids were co-transfected in 293T cells, followed by a co-immunoprecipitation assay. Proteins were immunoprecipitated from transfected cell lysates with either anti-Flag or anti-HA antibody, confirming the protein-protein interaction between LCE3D and SPRR2A or SPRR2F in cells (Figure 5D). Altogether, these data suggest that LCE is positively associated with SPRR.

\section{Drug Treatment Attenuated the Expression of SPRR and LCE in Psoriasis}

Subsequently, we constructed a model of IMQ-induced psoriasiform dermatitis in mice. Compared with the control group, the IMQ group showed obvious psoriasis-like dermatitis, thickened skin and psoriasis, while the skin lesions with tazarotene cream treatment significantly decreased (Figure 6A and B). We found that the expression level of SPRR2 and LCE3 genes were significantly increased in the IMQ group, but significantly attenuated in tazarotene cream treatment group (Figure 6C and D). As expected, the expression of these genes is down-regulated in psoriasis patients under clinical therapy (Figure S2). Therefore, the SPRR and LCE genes are closely associated with psoriasis.

\section{Discussion}

It is well known that psoriasis is a common multifactorial complicated skin disease. In the last decade, many studies have been carried out to investigate psoriasis caused by epidermal hyperproliferation and aberrant keratinocyte differentiation. ${ }^{31,32}$ However, the pathogenesis of aberrant epidermal keratinocyte differentiation is still ambiguous. In the present study, the gene expression profile GSE109248 was obtained from the GEO database to explore the DEGs in psoriasis. The DEGs were significantly enriched in keratinocyte differentiation, keratinization, and epidermis development by GO enrichment analysis. Interestingly, the SPRR and LCE genes were also identified as a module in the constructed PPI network. And the association of SPRR and LCE genes was further detected by correlation analysis.

It is reported that interactions between genes are associated with the pathogenesis of psoriasis. Gene-gene interactions in IL23/Th17 pathway contribute to psoriasis susceptibility in Chinese Han population. ${ }^{7,8}$ In particular, the interactions between late cornified envelope proteins and corneodesmosin were also analyzed. ${ }^{9}$ Recently, 
A

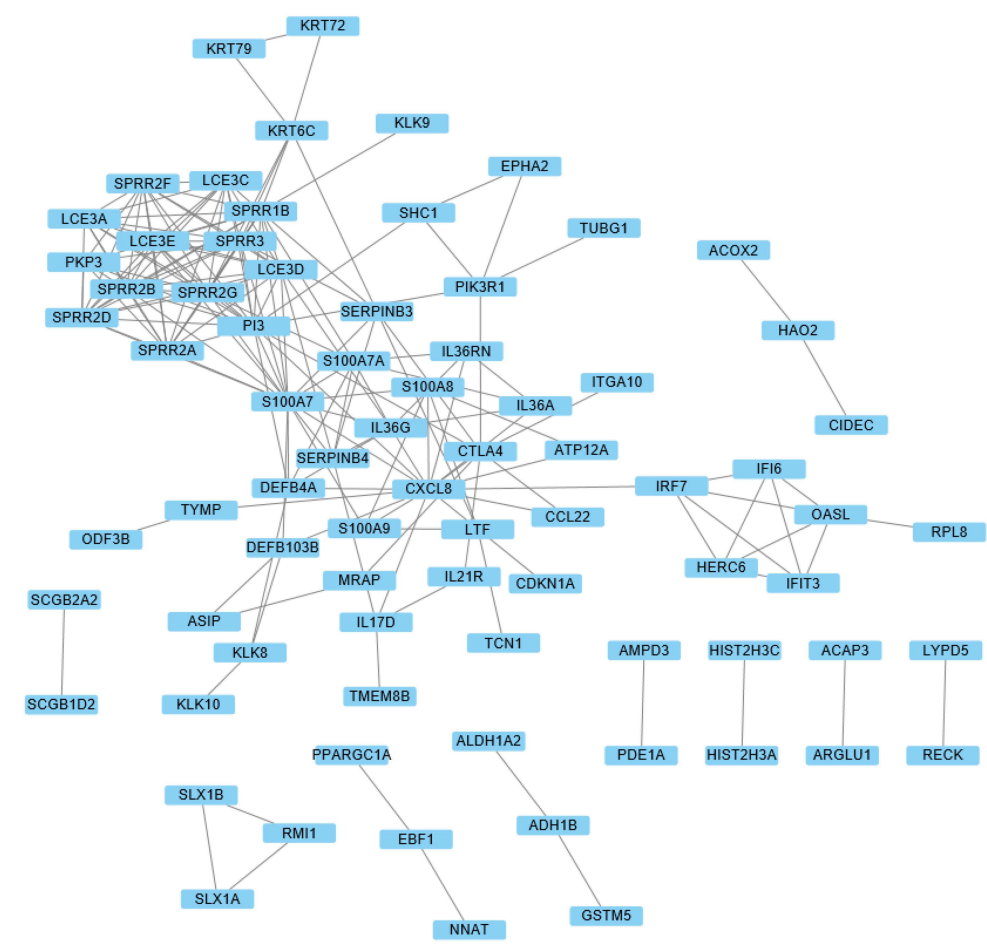

B

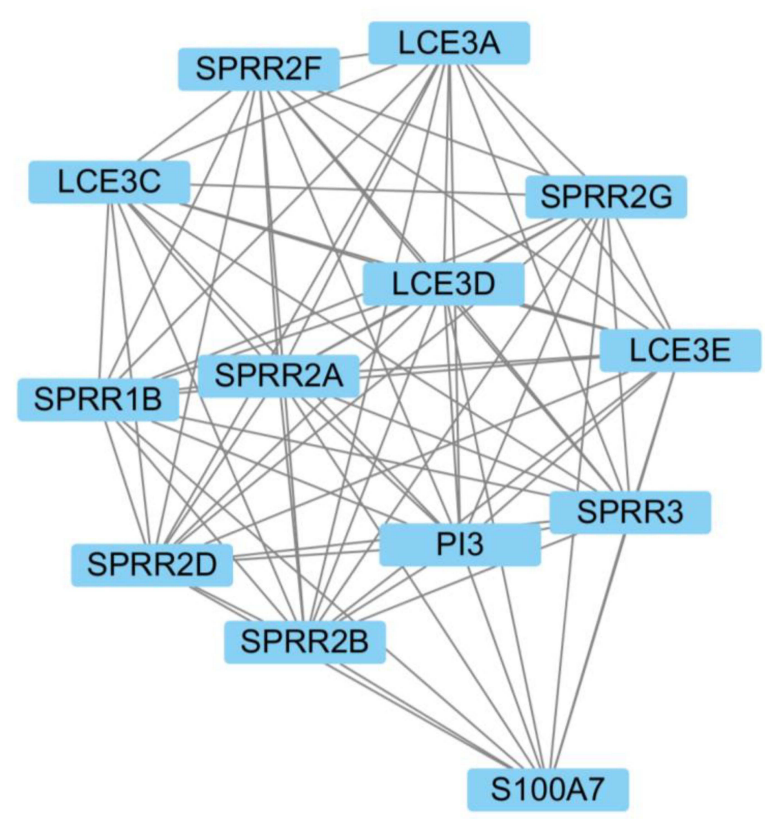

Figure 3 PPI network of differentially expressed genes. (A) PPI network of DEGs constructed by STRING and visualized using Cytoscape. (B) The significant module was identified from the PPI network.

stepwise regression analysis found that LILRB2 binds to HLA-B in two large psoriasis genome-wide association study cohorts totaling 10,069 subjects. ${ }^{10}$ However, there is no intuitive proof of these interactions. Likewise, although SPRR genes and LCE genes encode protein components of Epidermal Differentiation Complex and have been identified as risk factors for psoriasis patients, the association of SPRR and LCE genes has not been 
A
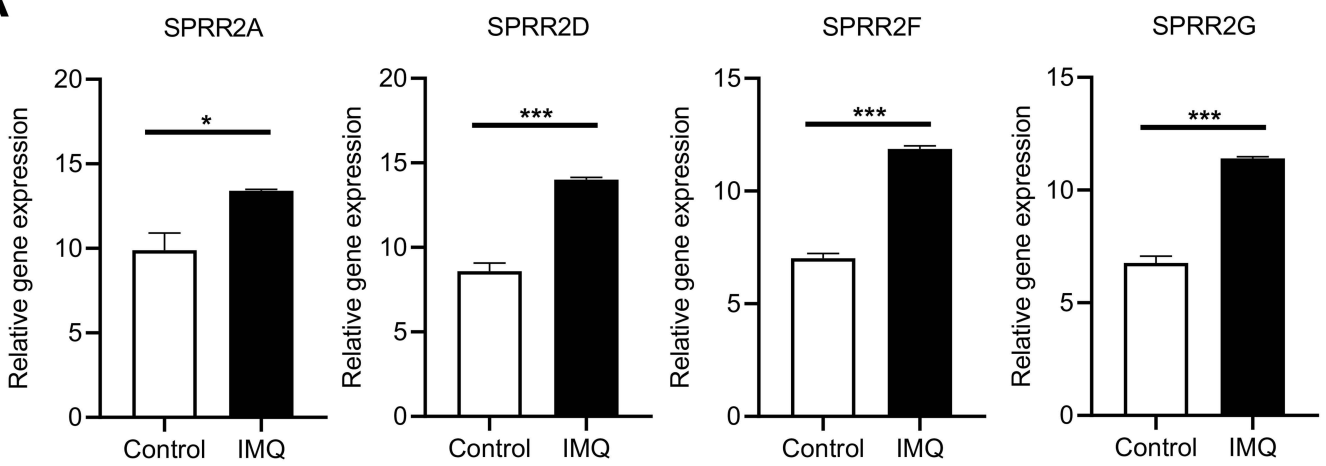

B

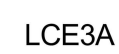

LCE3C

LCE3D
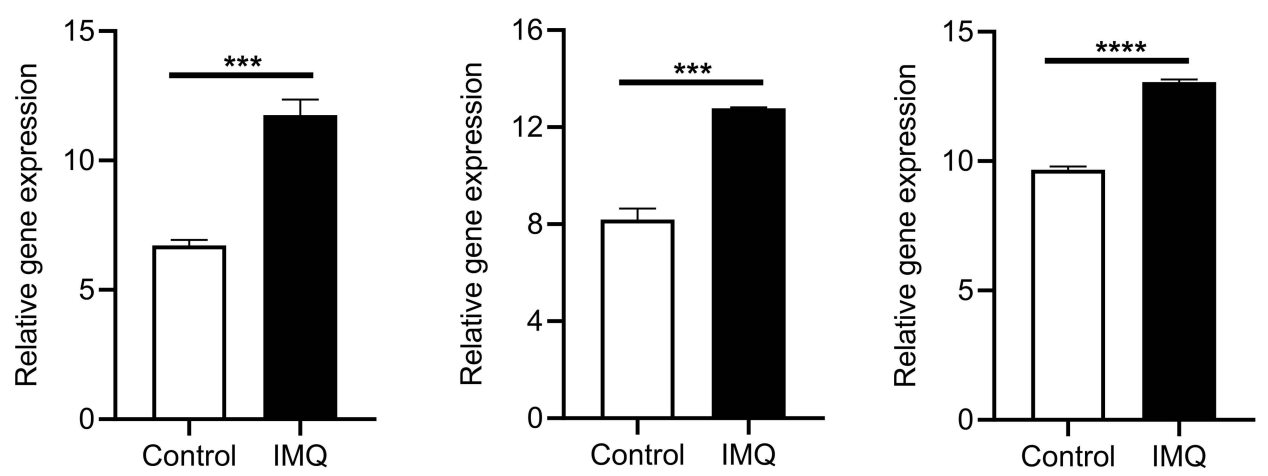

C

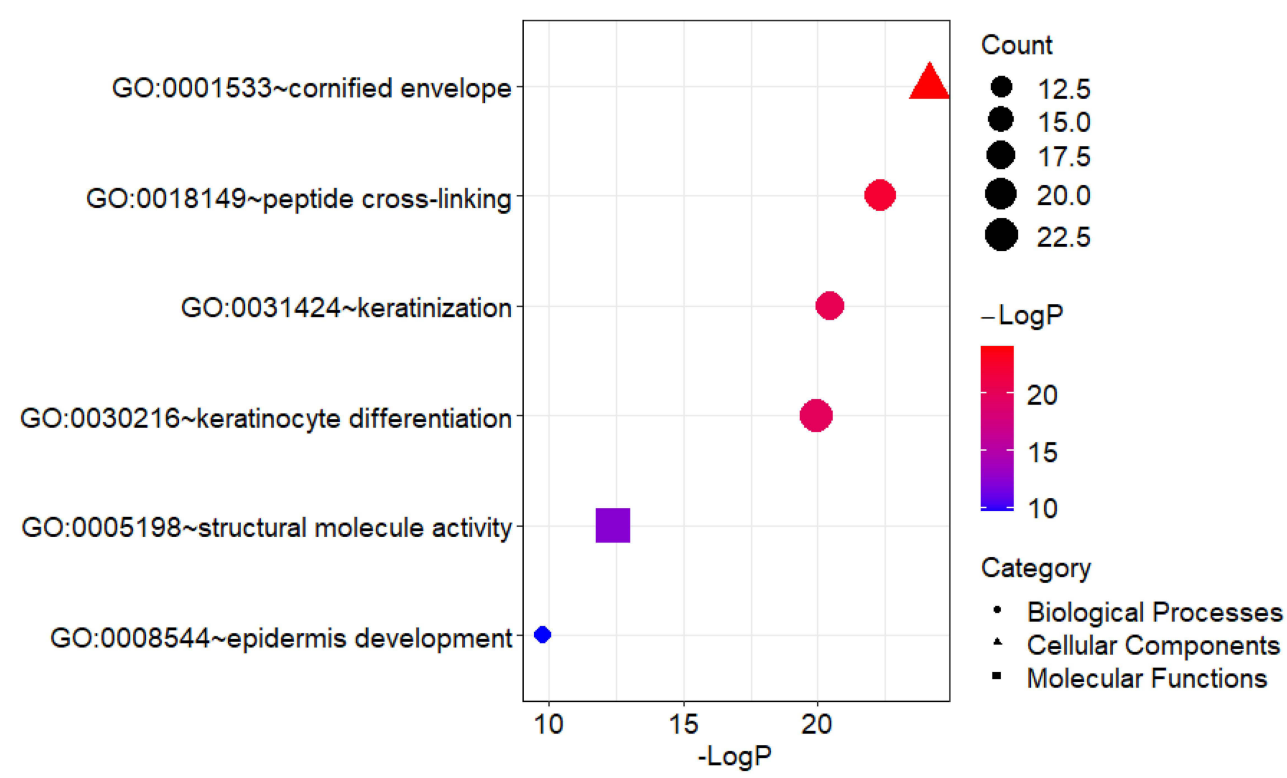

Figure 4 Validation of DEGs in psoriasis mice of dataset GSE63684. Expression levels of (A) SPRR and (B) LCE were significantly upregulated in psoriasis mice. *P $<0.05$, $* * * \mathrm{P}<0.001$ and $* * * * \mathrm{P}<0.0001$. (C) GO enrichment analysis of DEGs in psoriasis mice.

performed in-depth analysis in psoriasis. In our study, colocalization of SPRR2 and LCE3D was observed in 293 T cells by fluorescence microscopy. And their direct interactions are powerfully supported by the coimmunoprecipitation (CO-IP) results.
Further, a model of imiquimod (IMQ) induced psoriasiform dermatitis mice was constructed. The expression levels of SPRR and LCE genes were significantly increased in the IMQ-induced psoriasiform dermatitis mice but decreased comparatively in tazarotene cream 
A

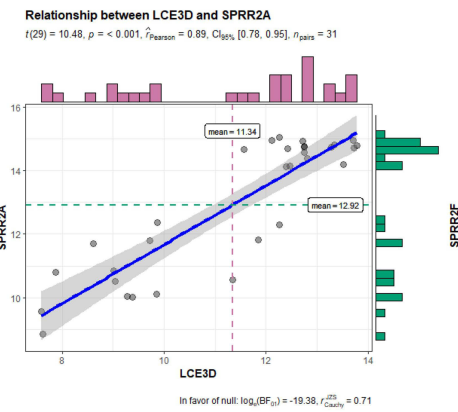

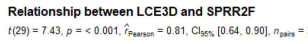

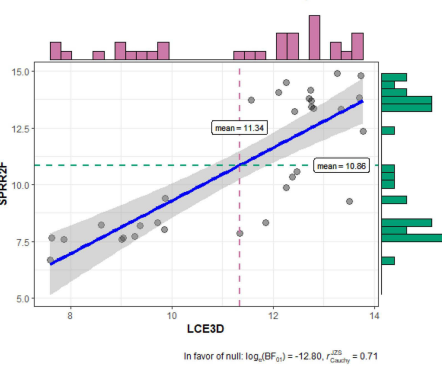

B

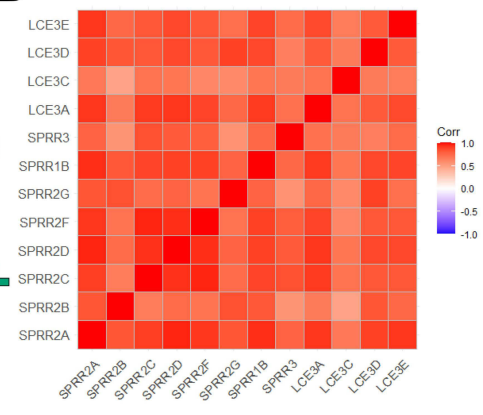

C
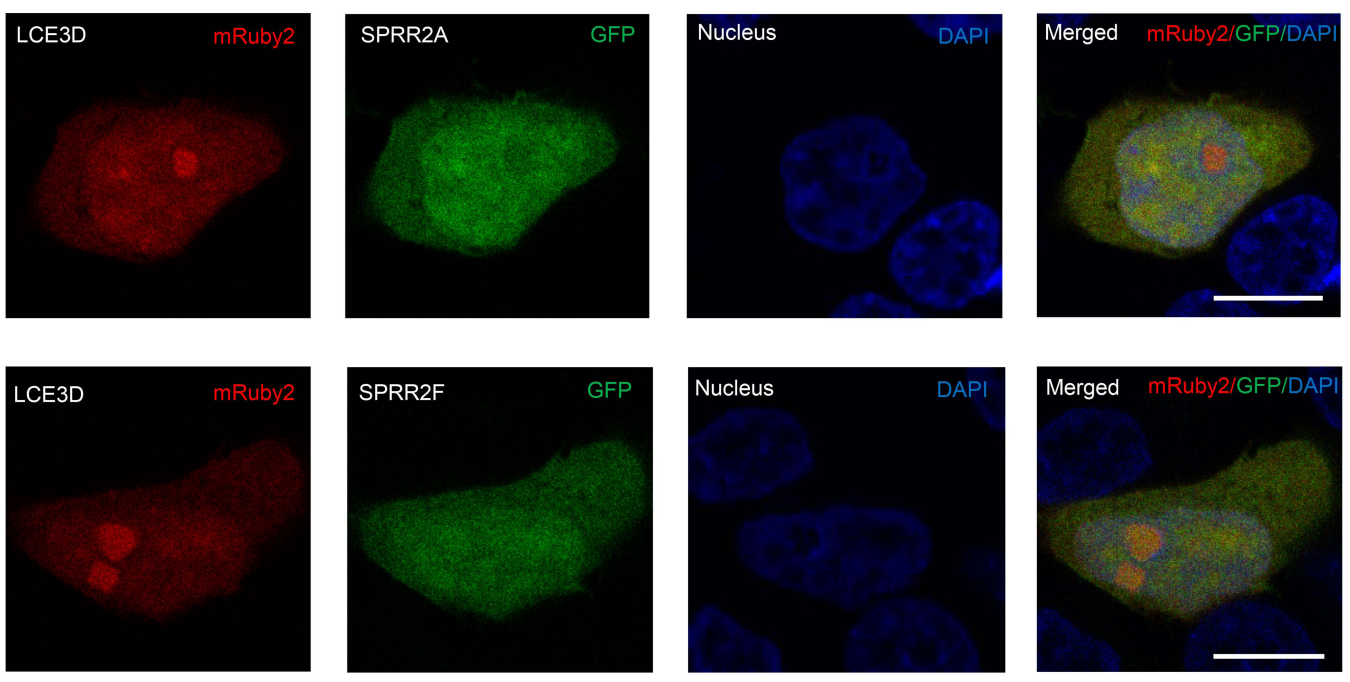

D

IP: HA Flag IgG

IB: HA

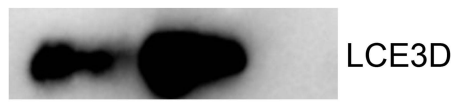

IB: Flag

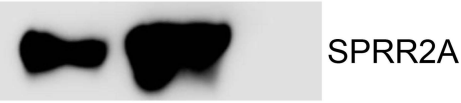

Input

IB: HA

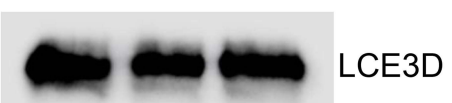

IB: Flag

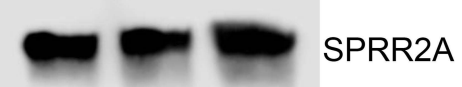

IP:

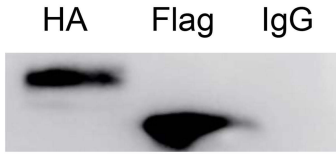

IB: HA

IB: Flag

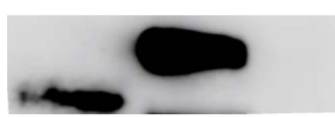

LCE3D

SPRR2F

LCE3D

Input

IB: HA

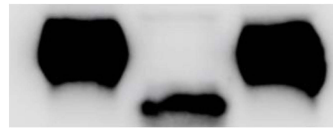

SPRR2F

LCE3D

IB: Flag

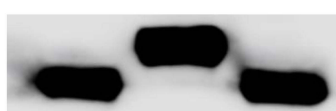

SPRR2F

Figure 5 The interaction of between SPRR and LCE. (A) The correlation between LCE3D and SPRR2. (B) The correlation between LCE and SPRR. (C) SPRR2 proteins colocalize with LCE3D upon co-expression in 293T cells. Nuclei were stained using 4',6-diamidino-2-phenylindole (DAPI). Scale bar represents I0 $\mu$ m. (D) Immunoassay of lysates of 293T cells transfected with plasmids encoding LCE3D and SPRR2A; LCE3D and SPRR2F, followed by immunoprecipitation (IP) with anti-HA, anti-Flag or the control antibody immunoglobulin $\mathrm{G}(\mathrm{IgG})$ and immunoblot analysis (IB) with anti-HA or anti-Flag.

treated mice. As expected, these genes are also downregulated in psoriasis patients under clinical therapy, indicating that the SPRR and LCEs were regulated simultaneously in psoriasis. And the functions of their interaction in psoriasis can be further explored because SPRR proteins and LCE proteins are not just the important 
A

Sham

IMQ

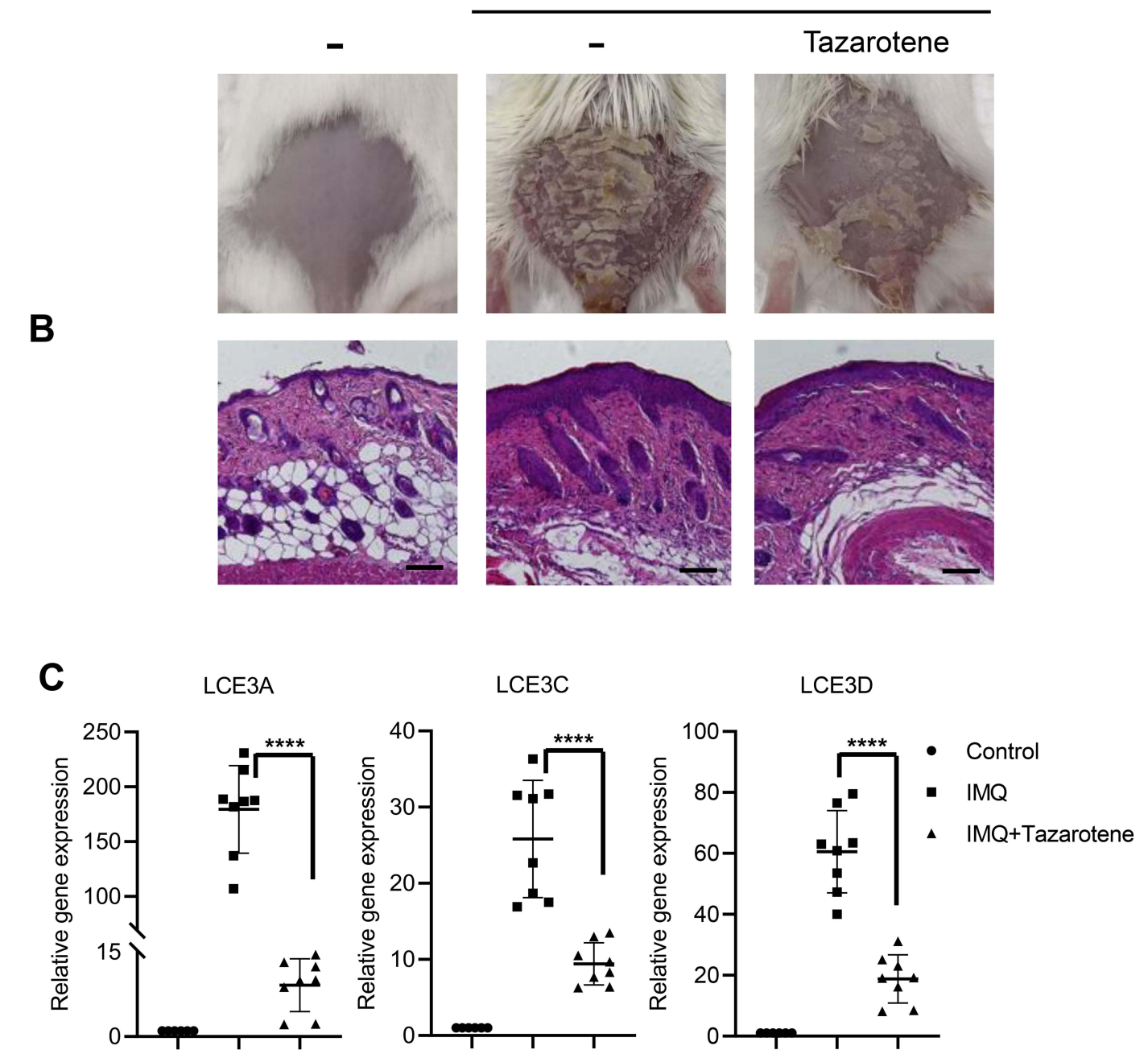

D

SPRR2A

SPRR2D

SPRR2F

SPRR2G
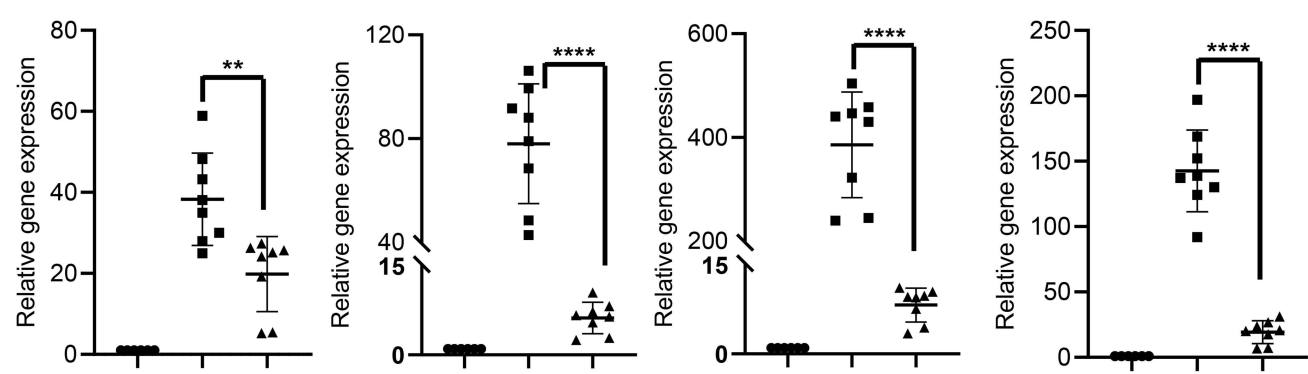

Figure 6 Expression of SPRR and LCE genes were significantly up-regulated in a model of psoriasiform dermatitis. (A) Photograph of mice back skin was taken on day 7. (B) H\&E staining of back skin sections were examined on day 7. Scale bar represents $50 \mu \mathrm{m}$. (C and D) SPRR and LCE gene expression levels in the skin lesions were evaluated by RT-qPCR. The results are presented as the means \pm standard deviation. All experiments were performed 3 times, and the representative results are presented. ** $<<0.0 \mathrm{I}$ and $* * * * P<0.0001$.

Abbreviation: IMQ, imiquimod.

components of cornified envelope precursor proteins, ${ }^{33}$ they also play roles in other processes, like SPRR proteins multimerized by cysteine oxidation. ${ }^{34}$ Further PPI analysis showed that some other proteins interact with SPRR and LCE, such as keratins, PI3, PKP3 and S100. Therefore, the interaction between SPRR and LCE may be a bridge for 
the association of psoriasis regulators, which needs to be further explored in the future.

Subsequently, in order to assess the possible biological consequences of the interaction between SPRR and LCE, we conducted correlation analysis in clinical psoriasis patients with different characteristics based on GEO datasets. Unexpectedly, the association between SPRR and LCE has no difference between mild psoriasis patients and severe psoriasis patients (Figure S4A), which suggests that the interaction of SPRR and LCE may be associated with the pathogenesis of psoriasis without affecting its development. However, the association between SPRR and LCE was weakened in psoriasis patients after guselkumab or ustekinumab treatment (Figure S4B and $\underline{\text { C). Thus, }}$ the extent of the association between SPRR and LCE may serve as a biomarker for psoriasis diagnosis.

\section{Conclusion}

We found that keratinocyte differentiation associated SPRR and LCE genes were identified as a module in the constructed PPI network. The association between SPRR and LCE genes was detected by correlation analysis. More importantly, the interactions between SPRR2 and LCE3D were further validated, and the SPRR and LCEs were regulated simultaneously in psoriasis. Additionally, the association between SPRR and LCE has no difference between mild psoriasis patients and severe psoriasis patients, but was weakened in psoriasis patients after guselkumab or ustekinumab treatment. In conclusion, the present study indicates that the interactions of SPRR proteins with LCE proteins may provide new insights into the pathogenesis of psoriasis.

\section{Ethics Statements}

All the animal experiments were approved by the Animal Ethics Committee of Xiamen University (acceptance no. XMULAC20170313). All animal experiments were performed in compliance with the guidelines from the Institutional Animal Care and Use Committee at Experimental Animal Centre in Xiamen University.

\section{Disclosure}

The authors declare no conflicts of interest.

\section{References}

1. Perera GK, Di Meglio P, Nestle FO. Psoriasis. Annu Rev Pathol. 2012;7(1):385-422. doi:10.1146/annurev-pathol-011811-132448
2. Nestle FO, Kaplan DH, Barker J. Psoriasis. N Engl J Med. 2009;361 (5):496-509. doi:10.1056/NEJMra0804595

3. Yamanaka K, Yamamoto O, Honda T. Pathophysiology of psoriasis: a review. J Dermatol. 2021;48(6):722-731. doi:10.1111/1346-8138.15 913

4. Takeshita J, Grewal S, Langan SM, et al. Psoriasis and comorbid diseases: epidemiology. J Am Acad Dermatol. 2017;76(3):377-390. doi:10.1016/j.jaad.2016.07.064

5. Michalek IM, Loring B, John SM. A systematic review of worldwide epidemiology of psoriasis. J Eur Acad Dermatol Venereol. 2017;31 (2):205-212. doi:10.1111/jdv.13854

6. Armstrong AW, Read C. Pathophysiology, clinical presentation, and treatment of psoriasis: a review. JAMA. 2020;323(19):1945-1960. doi:10.1001/jama.2020.4006

7. Wang WJ, Yin XY, Zuo XB, et al. Gene-gene interactions in IL23/ Th17 pathway contribute to psoriasis susceptibility in Chinese Han population. J Eur Acad Dermatol Venereol. 2013;27(9):1156-1162. doi:10.1111/j.1468-3083.2012.04683.x

8. Wang J, Ding Y, Fan X, et al. Gene-gene interaction between LCE and CLEC16A increases the risk of psoriasis in a Chinese population. Ann Dermatol. 2014;26(3):421-423. doi:10.5021/ad.2014.26.3.421

9. Bergboer JG, Dulak MG, van Vlijmen-Willems IM, et al. Analysis of protein-protein interaction between late cornified envelope proteins and corneodesmosin. Exp Dermatol. 2014;23(10):769-771. doi:10.11 11/exd. 12524

10. Yanovsky RL, Chen H, Leslie S, Carrington M, Liao W. The interaction of LILRB2 with HLA-B is associated with psoriasis susceptibility. J Invest Dermatol. 2020;140(6):1292-1295.e1293. doi:10.1016/j.jid.2019.12.006

11. Jia J, Li C, Yang J, et al. Yes-associated protein promotes the abnormal proliferation of psoriatic keratinocytes via an amphiregulin dependent pathway. Sci Rep. 2018;8(1):14513. doi:10.1038/s41598018-32522-y

12. Gruber R, Elias PM, Crumrine D, et al. Filaggrin genotype in ichthyosis vulgaris predicts abnormalities in epidermal structure and function. Am J Pathol. 2011;178(5):2252-2263. doi:10.1016/j.ajpath. 2011.01.053

13. Mischke D, Korge BP, Marenholz I, Volz A, Ziegler A. Genes encoding structural proteins of epidermal cornification and S100 calcium-binding proteins form a gene complex ("epidermal differentiation complex") on human chromosome 1q21. J Invest Dermatol. 1996;106(5):989-992. doi:10.1111/1523-1747.ep12338501

14. Henry J, Toulza E, Hsu CY, et al. Update on the epidermal differentiation complex. Front Biosci. 2012;17(1):1517-1532. doi:10.2741/ 4001

15. Hohl D, de Viragh PA, Amiguet-Barras F, Gibbs S, Backendorf C, Huber M. The small proline-rich proteins constitute a multigene family of differentially regulated cornified cell envelope precursor proteins. J Invest Dermatol. 1995;104(6):902-909. doi:10.1111/15231747.ep12606176

16. Gibbs S, Fijneman R, Wiegant J, van Kessel AG, van De Putte P, Backendorf C. Molecular characterization and evolution of the SPRR family of keratinocyte differentiation markers encoding small proline-rich proteins. Genomics. 1993;16(3):630-637. doi:10.1006/ geno.1993.1240

17. Shen C, Gao J, Yin X, et al. Association of the late cornified envelope-3 genes with psoriasis and psoriatic arthritis: a systematic review. J Genet Genomics. 2015;42(2):49-56. doi:10.1016/j.jgg.20 15.01.001

18. Carregaro F, Stefanini AC, Henrique T, Tajara EH. Study of small proline-rich proteins (SPRRs) in health and disease: a review of the literature. Arch Dermatol Res. 2013;305(10):857-866. doi:10.1007/ s00403-013-1415-9

19. Anthony RM, Brown TJ, French GL. DNA array technology and diagnostic microbiology. Expert Rev Mol Diagn. 2001;1(1):30-38. doi:10.1586/14737159.1.1.30 
20. Wang J. From DNA biosensors to gene chips. Nucleic Acids Res. 2000;28(16):3011-3016. doi:10.1093/nar/28.16.3011

21. Barrett T, Troup DB, Wilhite SE, et al. NCBI GEO: archive for high-throughput functional genomic data. Nucleic Acids Res. 2009;37(suppl_1):D885-890. doi:10.1093/nar/gkn764

22. Edgar R, Domrachev M, Lash AE. Gene expression omnibus: NCBI gene expression and hybridization array data repository. Nucleic Acids Res. 2002;30(1):207-210. doi:10.1093/nar/30.1.207

23. Ashburner M, Ball CA, Blake JA, et al. Gene ontology: tool for the unification of biology. The gene ontology consortium. Nat Genet. 2000;25(1):25-29. doi:10.1038/75556

24. Franceschini A, Szklarczyk D, Frankild S, et al. STRING v9.1: protein-protein interaction networks, with increased coverage and integration. Nucleic Acids Res. 2013;41(D1):D808-815. doi:10.1093/ nar/gks1094

25. Szklarczyk D, Franceschini A, Wyder S, et al. STRING v10: protein-protein interaction networks, integrated over the tree of life. Nucleic Acids Res. 2015;43(D1):D447-D452. doi:10.1093/nar/ gku1003

26. Shannon P, Markiel A, Ozier O, et al. Cytoscape: a software environment for integrated models of biomolecular interaction networks. Genome Res. 2003;13(11):2498-2504.

27. van der Fits L, Mourits S, Voerman JS, et al. Imiquimod-induced psoriasis-like skin inflammation in mice is mediated via the IL-23/IL17 axis. J Immunol. 2009;182(9):5836-5845. doi:10.4049/jimmunol. 0802999
28. Schukur L, Geering B, Charpin-El Hamri G, Fussenegger M. Implantable synthetic cytokine converter cells with AND-gate logic treat experimental psoriasis. Sci Transl Med. 2015;7(318):318ra201. doi:10.1126/scitranslmed.aac4964

29. Sobiak B, Graczyk-Jarzynka A, Leśniak W. Comparison of DNA methylation and expression pattern of S100 and other epidermal differentiation complex genes in differentiating keratinocytes. J Cell Biochem. 2016;117(5):1092-1098. doi:10.10 $02 /$ jcb. 25392

30. Holthaus KB, Lachner J, Ebner B, Tschachler E, Eckhart L. Gene duplications and gene loss in the epidermal differentiation complex during the evolutionary land-to-water transition of cetaceans. Sci Rep. 2021;11(1):12334. doi:10.1038/s41598-021-91863-3

31. Miyai M, Hamada M, Moriguchi T, et al. Transcription factor MafB coordinates epidermal keratinocyte differentiation. J Invest Dermatol. 2016;136(9):1848-1857. doi:10.1016/j.jid.2016.05.088

32. Lee SE, Lee SH. Skin barrier and calcium. Ann Dermatol. 2018;30 (3):265-275. doi:10.5021/ad.2018.30.3.265

33. Stawczyk-Macieja M, Szczerkowska-Dobosz A, Rębała K, PurzyckaBohdan D. Genetic background of skin barrier dysfunction in the pathogenesis of psoriasis vulgaris. Postepy Dermatol Alergol. 2015;32(2):123-126. doi:10.5114/pdia.2014.44003

34. Vermeij WP, Alia A, Backendorf C. ROS quenching potential of the epidermal cornified cell envelope. J Invest Dermatol. 2011;131 (7):1435-1441. doi:10.1038/jid.2010.433

\section{Publish your work in this journal}

Clinical, Cosmetic and Investigational Dermatology is an international, peer-reviewed, open access, online journal that focuses on the latest clinical and experimental research in all aspects of skin disease and cosmetic interventions. This journal is indexed on CAS.
The manuscript management system is completely online and includes a very quick and fair peer-review system, which is all easy to use. Visit http://www.dovepress.com/testimonials.php to read real quotes from published authors. 